\title{
Sensitivity analysis of the Expansion Process for Alloy UNS N08028
}

\author{
Aitor Navarro ${ }^{1, a}$, Mario Lechner ${ }^{1}$, Unai Ruiz ${ }^{1}$ and Alejandra Lopez ${ }^{1}$ \\ ${ }^{1}$ Tubacex Group, Innovation Department, C/ Tres cruces 8, 01400 Llodio, Alava - Spain
}

\begin{abstract}
Due to the good mechanical properties of forged parts, the forging process plays a decisive role in the manufacturing of seamless stainless steel pipes for oil country tubular goods (OCTG) lines. Tough competition between manufacturers gives them plenty of incentive to make their processes in raw material and energy usage more and more efficient. In this context the expansion process is one of the critical production steps in the manufacturing of seamless stainless steel pipes. This work presents a sensitivity analysis of a finite element method (FEM) for the simulation of the expansion of the alloy UNS N08028. The input parameters ram speed, tool angle, initial ID and final ID of the billet as well as temperature were used to describe responses like tool wear and material loss. With the aim to minimize the tool wear and to reduce the material waste, a study of influence of the input parameters on the mentioned responses were performed. This development is supported by experimental work in order to validate the simulation model. The sector demand for new materials with specific properties and the cost-intensive experimental trials justifies the use of such simulation tools and opens great opportunities for the industry.
\end{abstract}

\section{Introduction}

The extension of product portfolio with new grades and materials with specific properties demanded by the seamless stainless steel pipe sector significantly increases the number of tests in the production line of the companies. For this reason, a FEM model has been established as a highly valuable tool to detect process failures in early design stages and to reduce production standstill times.

Several techniques and processes exist in the field of metallurgy to produce high-alloyed seamless steel pipes, which were successfully developed in the 20th century. The extrusion process is one of these methods, which competes with different rolling processes, such as pierce rolling. The low billet weight and long dead cycle times are great disadvantages for the extrusion process but the low-cost of the tools gives an opportunity on small campaigns, for example, unusual materials or dimensions $[2,4]$.

Round billets are usually used for the extrusion process. When high-alloyed steel pipes are manufactured, the billet is normally drilled, heated and the bore is expanded to a desired diameter [2]. The following picture shows an example of a typical manufacturing process chain for seamless pipe production.

The expansion process is a well-known method to produce expanded billet in order to reduce material waste during the pipe production. In this step, the billet is introduced in a container and compressed with an expansion tool - a so-called nose - characterised by its inclined contact area.

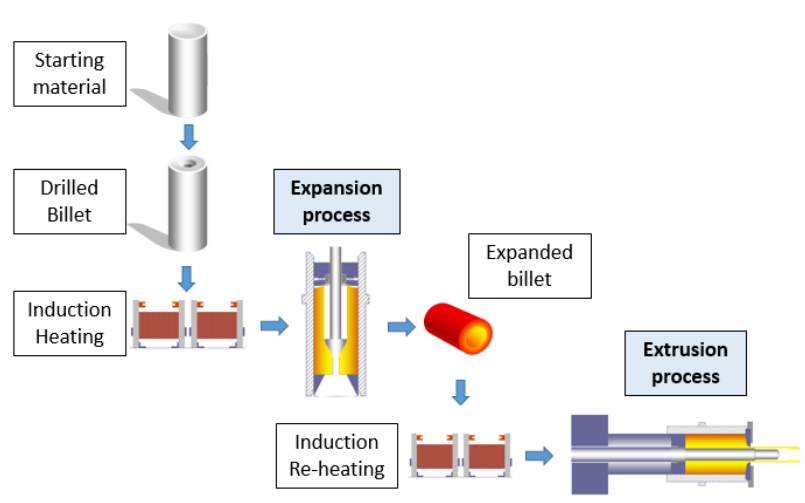

Figure 1. Sketch of seamless pipe manufacturing process chain.

As a result, the material flows along this tool expanding the inner diameter of the billet. The expansion process ends by cutting of a piece of material. This material loss - known as remainder - and its weight depends on the tool design, temperature of the billet, lubrication, tool velocity, material type and the expansion limit. The picture of Figure 2 shows a sketch of the expansion process [4].

Seamless stainless steel tubes are used in high resistance corrosion environments present in the oil and gas industry, the chemical sector, the mechanical engineering industry and the nuclear industry. One of these high corrosion resistance materials is the alloy UNS N08028 and therefore this grade has been chosen to carry out this study. The main application area of alloy UNS N08028 is the oil and gas industry for OCTG lines [1].

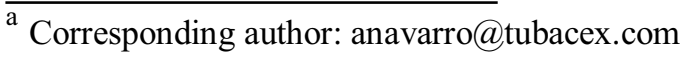




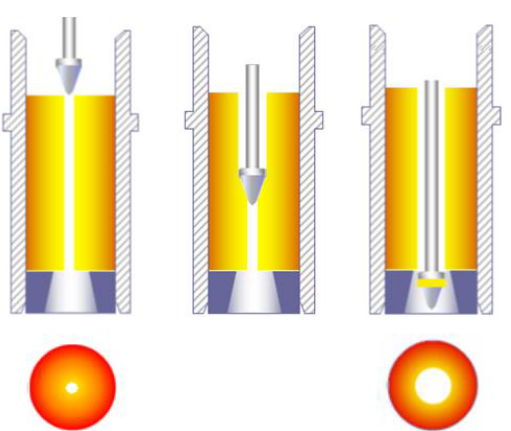

Figure 2. Sketch of expansion process steps.

For this reason, Tubacex developed a simulation model for the expansion process technology using alloy UNS N08028. This work focuses on increasing the tool life and reducing the material waste based on sensitivity analysis of the input parameters.

\section{Model validation}

\subsection{Simulation model}

In order to guarantee reliability of the results obtained from simulation, it is necessary to make a comparison with experimental results. Thus, a model validation was carried out first. Figure 3 shows the comparison between the experimental process and the simulation process.

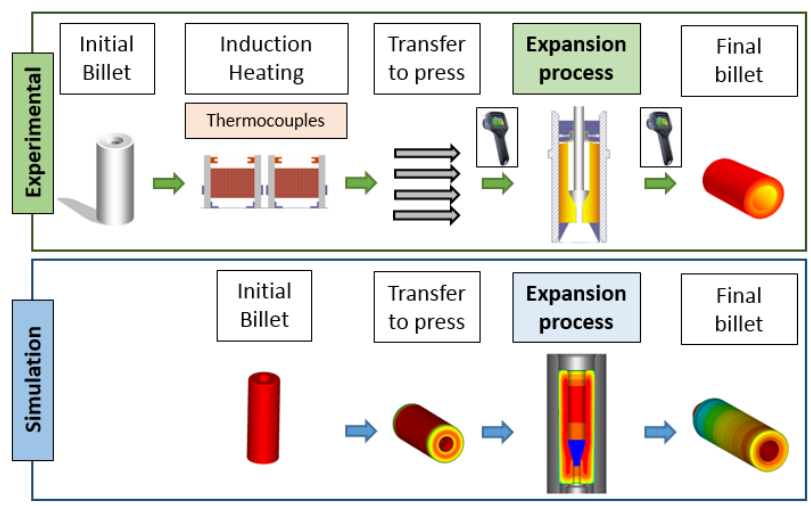

Figure 3. Simulation steps comparing with experimental steps.

Regarding the simulation, the commercial software FORGE NxT1.0 was chosen for this research project. High-performance workstation with Intel Xeon CPU E5-2643v2 processor was used. The uniaxial hot tensile test was selected to describe the alloy UNS N08028 deformation behaviour [3]. The test was carried out at temperatures between $900-1220^{\circ} \mathrm{C}$ and strain rates between $0.1-10 \mathrm{~s}^{-1}$. The temperature was obtained with thermocouples, and the strain and strain rate was measured with Gom Aramis method. The Hansel-Spittel approach was applied to extend the range of the flow stress curve. The material data was implemented in the database of the software with Point-by-point method. A 2D thermo-mechanical model was used in order to reduce the computation time. Glass was used as the lubrication medium and plays an important role for this type of processes. Its friction coefficient and heat exchange coefficient at a temperature of $1200^{\circ} \mathrm{C}$ are $0.03-0.13$ and $1500-2200 \mathrm{~W} / \mathrm{m}^{2} \mathrm{~K}$, respectively $[4,6,7]$. Small variations in the friction and heat exchange coefficient give significant differences in expansion press load and billet temperature.

\subsection{Experimental results}

As for the experimental work, two trials were conducted for model validation. Furthermore, one billet was prepared to extract the temperature data from the induction heating system with five thermocouples placed inside. The experimental results reveal the homogeneity of the temperature achieved during the induction heating process. Even so few differences were found between bottom and top, and between inner and outer side of the billet.

After the induction heating process, the billets were transferred to the expansion press. With help of a thermographic camera, the thermal image was captured before the expansion of the billet and at the end of the expansion process. The following graphic shows the temperature comparison between experimental and numerical results before the billet is set into the container.

\section{Temperature comparison before setting into container}

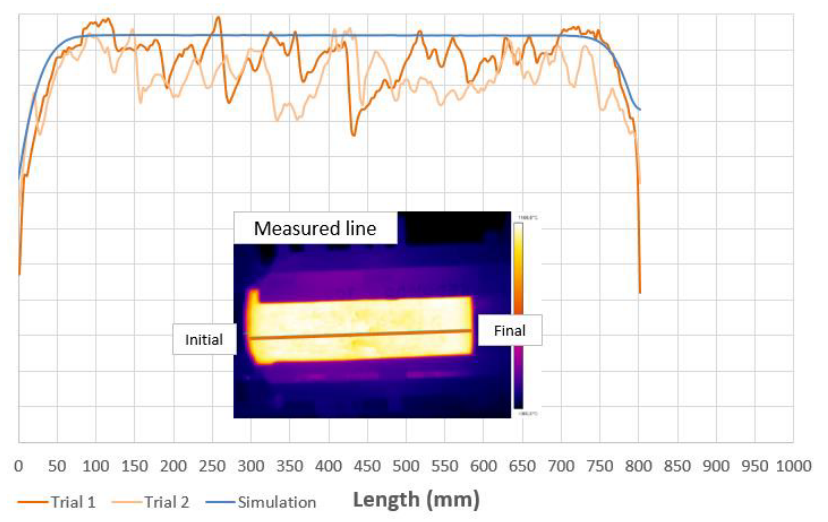

Figure 4. Temperature comparison between experimental and simulation just before the billet is set into the container.

Figure 4 shows oscillations in the experimental temperature data caused by the glass coating. Despite this fact, good agreement can be observed between experimental and numerical results.

The temperature comparison between experimental and numerical results at the end of the expansion can be seen in figure 5 .

The results show a difference between experimental and numerical model of approximately $70^{\circ} \mathrm{C}$ at the left side of the billet. Extensive temperature testing was carried out to determine the veracity of the temperature curve obtained in the simulation. The collected results revealed that the glass coating had a great effect on the measurements obtained with the thermographic camera. Thus, care must be taken when using an infrared radiation system to measure the temperature on glass-lubricated billets. 


\section{Temperature comparison after expansion process}

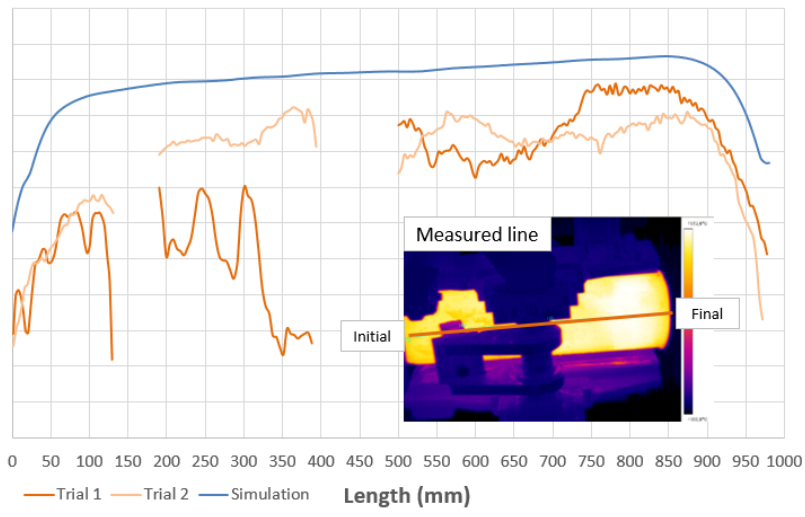

Figure 5. Temperature comparison between experimental and simulation after the expansion process.

The expansion process was carried out with a hydraulic press and with constant ram speed. The expansion press load was measured. The following diagram shows a comparison between experimental and numerical load.

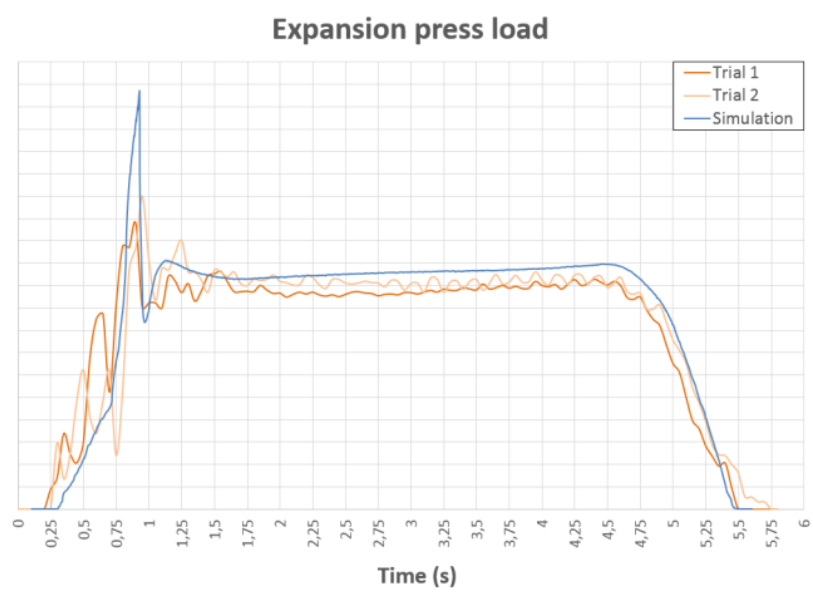

Figure 6. Load comparison between experimental and numerical results during the expansion process.

As can be seen, the result of the numerical model shows a good agreement with the experiment. The average value of the simulation load in the steady state is approx. 3-5\% higher than the experimental load. Regarding the graphic analysis, two different forging behaviours can be clearly distinguished: At the beginning of the process, the press compresses the billet and material does not flow alongside the expansion nose; as a result, high peaks of load are observed. When the billet contacts with the cutting ring in the lowest part, the material starts to flow, the load drops and a steady state is attained. If the material does not flow before the bore hole of the billet is closed, the tool is trapped inside and it can cause a fracture of the mandrel. A rise on the heat exchange results in an increase on the gradient of the load, and an increase of friction reveals an increase on the global load.

\subsection{Simulation results}

During the expansion process, the expansion nose compresses the billet and the material flows along the tool surface. As a result, the temperature increases inside the billet due to the deformation heating. This leads to a rise in the temperature difference between the inner and outer surface. The following figure shows the temperature evolution during the expansion process.

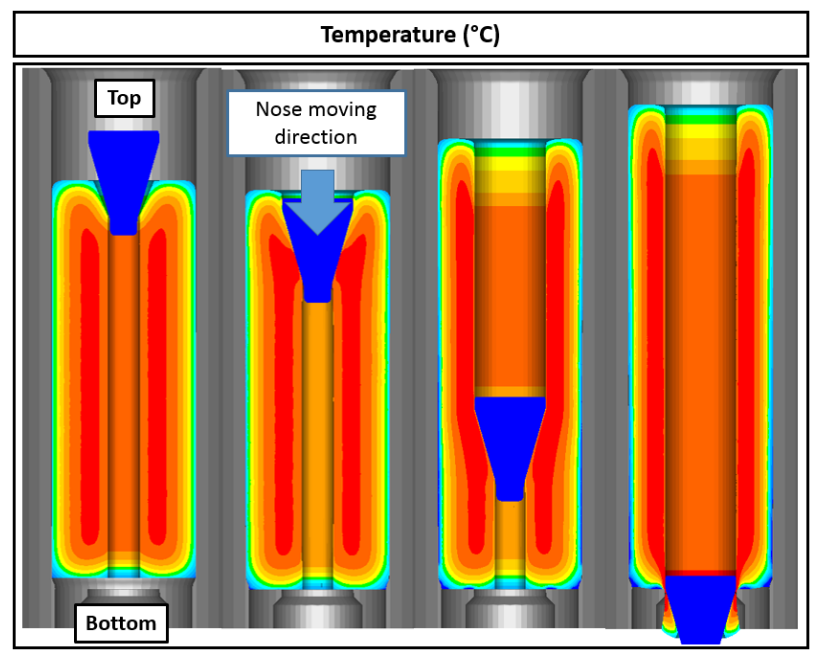

Figure 7. Temperature evolution during the expansion process.

As for deformation, high-accumulated strain values were obtained at the inside of the billet whereas it decreased towards the outside of the billet. Figure 8 shows the strain inside the billet obtained from simulation.

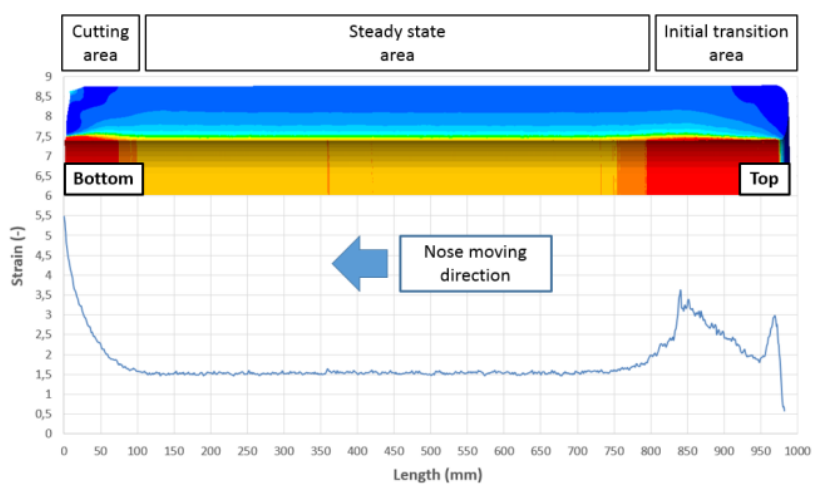

Figure 8. Accumulated strain inside the billet.

Regarding the strain in the inner surface, three areas can be clearly distinguished: an initial transition area with high level of strain due to the initial compression, a steady state with a constant strain of 1.5 and a cutting area with high strain values. This strain behaviour coincides with observations made in optical micrographs images.

The strain rate revealed the areas where significant deformation was located. The following picture shows the strain rate near the nose in the steady state.

According to the figure 9, the highest strain rate values are concentrated near the tool and two remarkable peaks can be detected. Hence, the deformation is located on the inner side of the billet. 


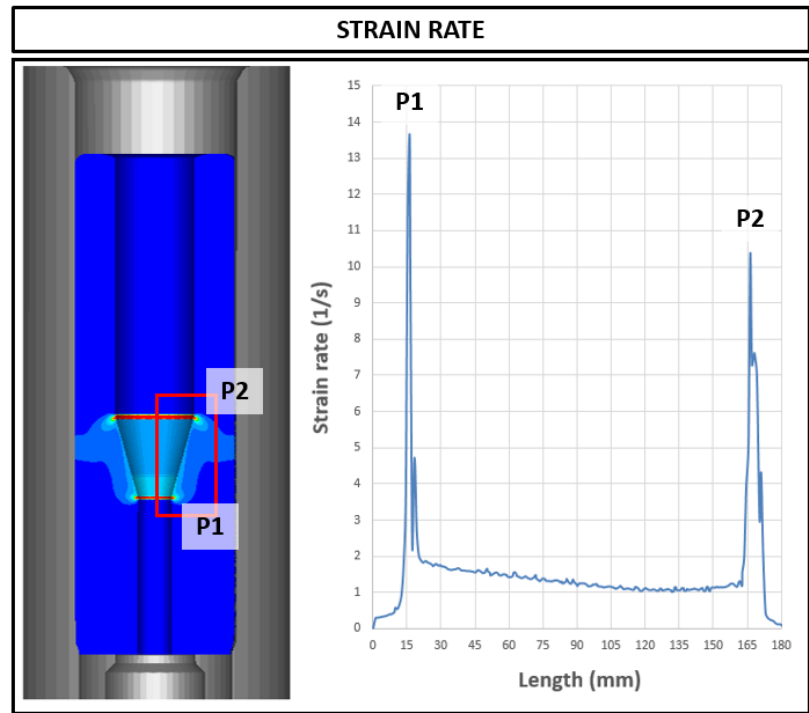

Figure 9. Strain rate near the nose in the steady state.

Regarding the defect predictions the Latham and Cockroft normalized criterion was applied. This model is used for cases with tensile failure.

$$
C_{C R}=\int_{0}^{\overline{\varepsilon_{f}}}\left(\frac{\sigma_{\max }}{\bar{\sigma}}\right) d \bar{\varepsilon}
$$

where $\sigma_{\max }$ is the maximum principal stress, $\overline{\varepsilon_{f}}$ is the strain at fracture, $\bar{\varepsilon}$ is the equivalent strain and $\bar{\sigma}$ is the equivalent stress. This criterion predicts defects when the material is under tensile stresses [3,5,8]. In accordance with this, the following picture shows the areas where the billet is prone to defects caused by the process.

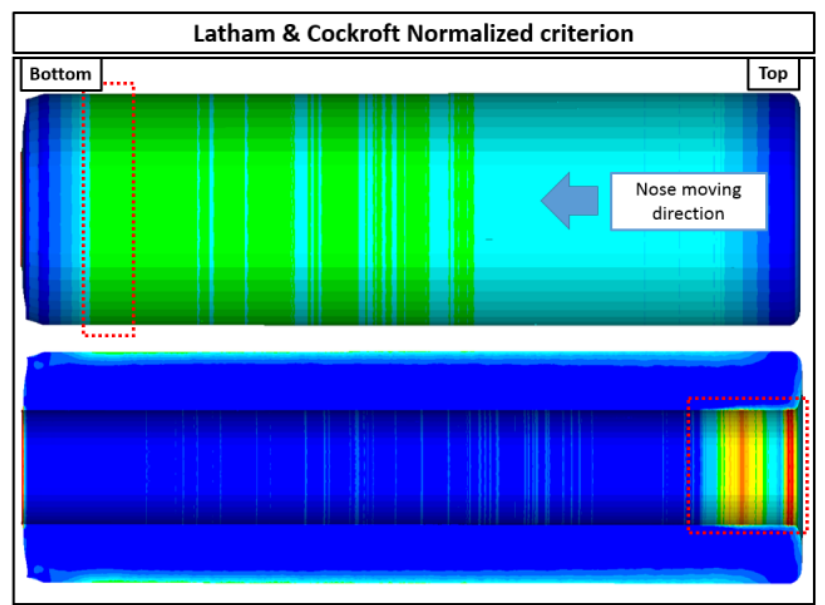

Figure 10. Damage critical areas predicted by simulation.

The simulation results shown in figure 10 reveal two critical areas; the initial contact area between the nose and the billet, and the external surface on the bottom side of the billet.

Figure 11 shows defects after pickling process. This figure reveals a surface defect in the bore hole at the top of the billet and scratches at the edge of the top, which agrees well with the simulation results. The experimental results carried out did not show defects at the external surface, although long-time experience specifies this as a critical area. High friction values or low billet temperature can cause cracks in this area.

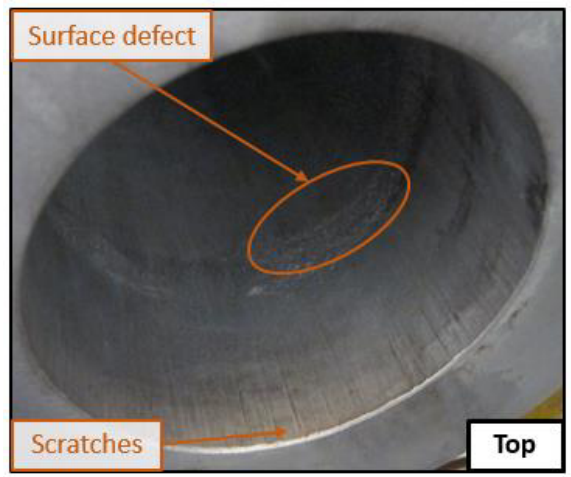

Figure 11. Defects in the top side after pickling process.

\section{Sensitivity Analysis}

With the help of validation work, a sensitivity analysis was carried out and critical parameters of the process were defined [4]. Some of these are the nose angle, the ram speed, the billet initial ID, the billet final ID and the temperature. To define the upper and lower limits of the input parameters some previous simulations were performed first.

Afterwards, a DOE for the simulation with the above five parameters was created using the software MINITAB, which was also used to analyse the sensitivity study [9]. Two main objectives were determined for the process optimisation: the reduction of the tool costs and the reduction of the raw material costs. Thus, the tool wear (TW) and the total material waste (TMW) were fixed as measurable output data.

Regarding the reduction of the tool costs, the maximum value of the TW was chosen for the sensitivity analysis. The results are shown in the following figure.

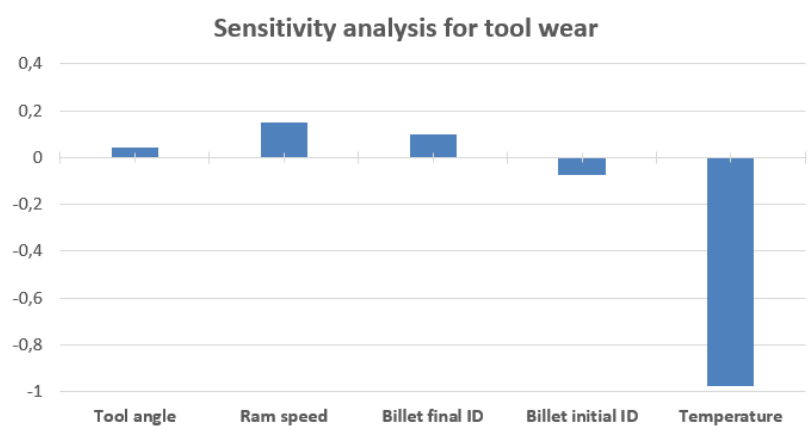

Figure 12. Sensitivity analysis for the TW.

The sensitivity analysis reveals the influence of the input parameters on the output. As can be clearly seen, the billet temperature has the strongest influence on the response of the TW. The ram speed and the billet final ID have a weak influence, whereas the nose angle and the billet initial ID do not show any relevant influence on TW.

According to the results aforementioned, a multiple linear model was found with the most important input parameters. These parameters were defined with help of 
the size of the $\mathrm{R}^{2}$-value. The accuracy of the obtained model is approx. 97\%. The following graphic shows the result.

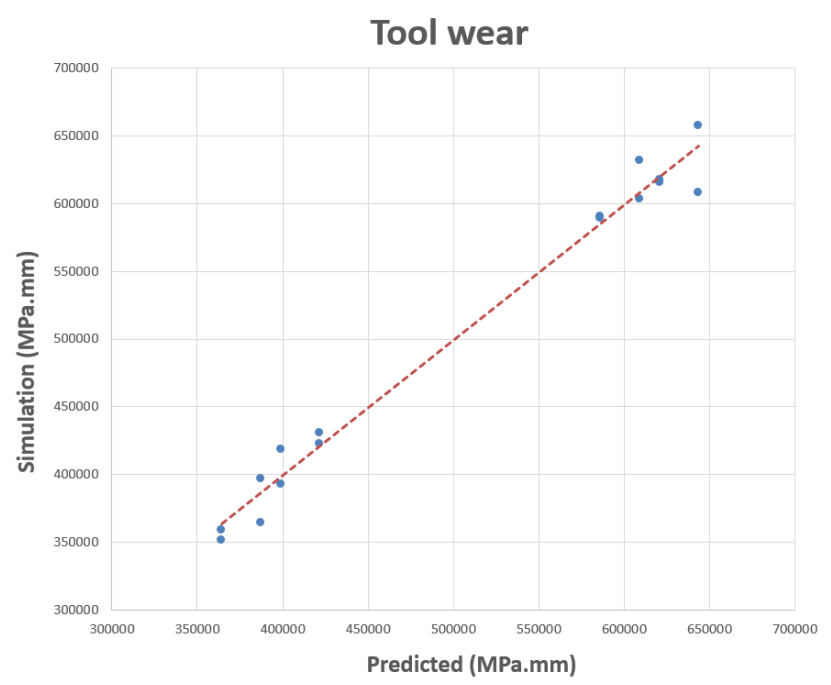

Figure 13. Predicted tool wear using multiple linear model compared to simulation results.

As for the reduction of the raw material costs, the maximum value of the TMW was chosen to carry out the sensitivity analysis using a multiple linear model. The TMW was defined as a combination of machining and remainder losses. The results are shown in the next figures.

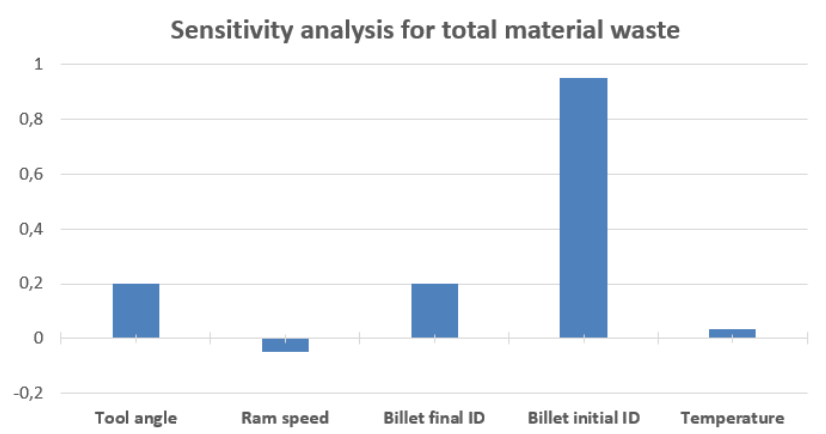

Figure 14. Sensitivity analysis for the total material waste.

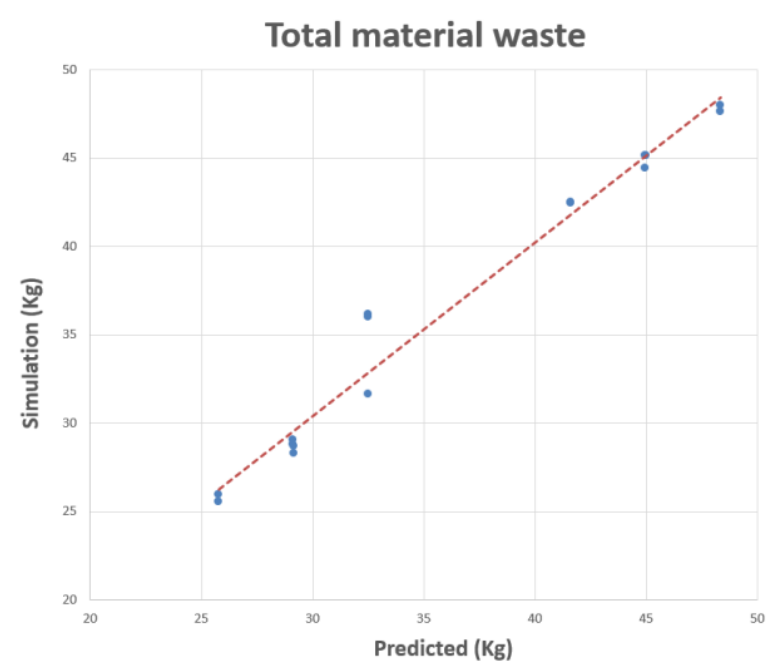

Figure 15. Predicted TMW using multiple linear model compared to simulation results.
As shown in figure 14, the billet initial ID has the strongest impact on the response of the TMW. The tool angle and the billet final ID have a weak influence, while the ram speed and the temperature do not show any relevant influence on TMW.

With the help of the relevant input parameters, a model with an accuracy of about $97 \%$ can be found. The result is shown in the following figure.

\section{Conclusions}

A simulation model for the expansion process was developed for alloy UNS N08028. The study showed good agreement between simulation and experimental results. Critical process parameters like friction, heat exchange, tool angle, ram speed, billet final ID, billet initial ID and temperature were identified. The model was able to predict with an acceptable accuracy defects, expansion press load and material wastes. High deformation areas were detected inside the billet with $150 \%$ of accumulated strain, whereas the accumulated strain at the outside was less than $30 \%$. Two critical areas were detected where the billet is prone to defects.

Sensitivity analysis was carried out to reduce the tool costs and the raw material costs. In relation with the first target, the billet temperature was detected as the strongest influence parameter on the response of the TW, whereas the tool angle and the billet initial ID did not show any relevant influence. As for the second target, the billet initial ID showed the strongest influence on the response of the TMW, while the ram speed and the temperature did not show any relevant influence. For the two objectives, models were found with the most important input parameters and the error in both cases was less than $3 \%$.

The study showed the capacity to predict defects using FEM and revealed the great possibilities that this type of method offers for the sensitivity analysis.

As for the future, the results of this study will be used to optimise the expansion process.

\section{Acknowledgment}

This work was supported by Tubacex Group. The author gratefully acknowledge the financial support from Tubacex Group.

\section{References}

1. https://www.tubacex.com

2. K. H. Brensing, B. Sommer, Res. Dpt., Salzgitter Mannesmann Röhrenwerke $\mathrm{GmbH}$, Mülheim an der Ruhr, Germany, (2009)

3. S. Fanini, Ph.D. diss., Dpt. of Mech. Inn. and Mgmt., Univ. of Padova, Padova, Italy, (2008)

4. S. Hansson, Ph.D. diss., Dpt. of Appl. Ph. of Mech. Eng., Luleå Univ. of Tech., Luleå, Sweeden, (2009) 
5. G. E. Dieter, H. A. Kuhn, S. L. Semiatin, Handbook of workability and process Design, ASM Inter., Mat. Park, Ohio, (2003)

6. J. Sejournet, J. Delcroix, l. Lubr. Eng. 11, 389-396, (1995)

7. C. Liu, L. Zhang, F. Kang, Adv. M. Res. 668, 856860 (2013)

8. M. G. Cockroft, D. J. Latham, J. of the Inst. of Me. 96, 33-39, (1996)

9. L. Eriksson, E. Johansson, N. Kettaneh-Wold, CA. Wikstrom, S.Wold, Design of ExperimentsPrinciples and Applications. Umetrics AB, (2008) 